\title{
ON A CONJECTURE BY KARLIN AND SZEGÖ
}

\author{
DEOK H. KIM AND KIL H. KWON \\ (Communicated by Hal L. Smith)
}

\begin{abstract}
In 1961, Karlin and Szegö conjectured : If $\left\{P_{n}(x)\right\}_{n=0}^{\infty}$ is an orthogonal polynomial system and $\left\{P_{n}^{\prime}(x)\right\}_{n=1}^{\infty}$ is a Sturm sequence, then $\left\{P_{n}(x)\right\}_{n=0}^{\infty}$ is essentially (that is, after a linear change of variable) a classical orthogonal polynomial system of Jacobi, Laguerre, or Hermite. Here, we prove that for any orthogonal polynomial system $\left\{P_{n}(x)\right\}_{n=0}^{\infty},\left\{P_{n}^{\prime}(x)\right\}_{n=1}^{\infty}$ is always a Sturm sequence. Thus, in particular, the above conjecture by Karlin and Szegö is false.
\end{abstract}

\section{INTRODUCTION}

At the end of their work [5, p.156], Karlin and Szegö made three conjectures for the characterization of classical orthogonal polynomials. The first and the third are answered by Al-Salam and Chihara [1] and Hahn [4] respectively. The second conjecture asks : if $\left\{P_{n}(x)\right\}_{n=0}^{\infty}$ is an orthogonal polynomial system and $\left\{P_{n}^{\prime}(x)\right\}_{n=1}^{\infty}$ is a Sturm sequence, then is $\left\{P_{n}(x)\right\}_{n=0}^{\infty}$ one of the three classical orthogonal polynomials of Jacobi $\left\{P_{n}^{(\alpha, \beta)}(x)\right\}_{n=0}^{\infty}(\alpha, \beta>-1)$, Laguerre $\left\{L_{n}^{(\alpha)}(x)\right\}_{n=0}^{\infty}(\alpha>-1)$, and Hermite $\left\{H_{n}(x)\right\}_{n=0}^{\infty}$ (possibly after a suitable linear change of variable)?

Conversely, it is well known that any orthogonal polynomial system $\left\{P_{n}(x)\right\}_{n=0}^{\infty}$ is a Sturm sequence (see Chihara [2], Chapter 1.5) and if $\left\{P_{n}(x)\right\}_{n=0}^{\infty}$ is a classical orthogonal polynomial system, then $\left\{P_{n}^{\prime}(x)\right\}_{n=1}^{\infty}$ is also a classical orthogonal polynomial system (known as the Hahn-Sonine theorem) and so is also a Sturm sequence.

We will show that for any orthogonal polynomial system $\left\{P_{n}(x)\right\}_{n=0}^{\infty},\left\{P_{n}^{\prime}(x)\right\}_{n=1}^{\infty}$ is always a Sturm sequence (but is not necessarily orthogonal). In particular, the answer to the above question by Karlin and Szegö is no.

The orthogonality considered in Karlin and Szegö [5] is the one with respect to a positive Stieltjes measure $d \mu(x)$, where $\mu(x)$ is a non-decreasing function. Here we consider a general sense of orthogonality with respect to a signed Stieltjes measure $d \mu(x)$, where $\mu(x)$ is a function of bounded variation.

\section{MAin RESUlts}

All polynomials in this work are assumed to be real polynomials in one variable. We use $\operatorname{deg}(P)$ to denote the degree of a polynomial $P(x)$ with the convention that $\operatorname{deg}(0)=-1$.

Received by the editors August 12, 1994.

1991 Mathematics Subject Classification. Primary 33C45, 42C05.

(C)1996 American Mathematical Society 
Definition 2.1 (Karlin and Szegö [5]). A sequence of polynomials $\left\{P_{n}(x)\right\}_{n=0}^{\infty}$ with $\operatorname{deg}\left(P_{n}\right)=n, n \geqslant 0$, is called a Sturm sequence on an open interval $I=$ $(a, b),-\infty \leq a<b \leq \infty$ if

(i) each $P_{n}(x)$ has exactly $n$ simple real zeros in $I$;

(ii) for each $n \geqslant 1$, zeros of $P_{n}(x)$ and $P_{n+1}(x)$ strictly interlace.

If $x_{n 1}<x_{n 2}<\cdots<x_{n n}$ are zeros of $P_{n}(x), n \geqslant 1$, then the above condition (ii) means

$$
x_{n+1, i}<x_{n i}<x_{n+1, i+1}, \quad 1 \leq i \leq n .
$$

In the following, we call a sequence of polynomials $\left\{P_{n}(x)\right\}_{n=0}^{\infty}$ a polynomial system (PS) if $\operatorname{deg}\left(P_{n}\right)=n, n \geqslant 0$.

Definition 2.2. A PS $\left\{P_{n}(x)\right\}_{n=0}^{\infty}$ is called a Tchebychev polynomial system (TPS) (respectively, an orthogonal polynomial system (OPS)) if there is a function $\mu(x)$ of bounded variation (respectively, a non-decreasing function $\mu(x)$ ) such that

$$
\int_{-\infty}^{\infty} P_{m}(x) P_{n}(x) d \mu(x)=K_{n} \delta_{m n}, \quad m \text { and } n \geqslant 0,
$$

where $K_{n} \neq 0$ (respectively, $\left.K_{n}>0\right), n \geqslant 0$.

We first find sufficient conditions for any given PS $\left\{P_{n}(x)\right\}_{n=0}^{\infty}$ under which both $\left\{P_{n}(x)\right\}_{n=0}^{\infty}$ and $\left\{P_{n}^{\prime}(x)\right\}_{n=1}^{\infty}$ are Sturm sequences.

Theorem 2.1. Let $\left\{P_{n}(x)\right\}_{n=0}^{\infty}$ be a PS such that all zeros of $P_{n}(x), n \geqslant 1$, are real and lie in $I=(a, b)$. Let

$$
W_{n}(x)=P_{n}(x) P_{n+1}^{\prime}(x)-P_{n}^{\prime}(x) P_{n+1}(x), \quad n \geqslant 0,
$$

be the Wronskian determinant of $P_{n}(x)$ and $P_{n+1}(x)$. If $W_{n}\left(x_{0}\right) W_{n}\left(x_{1}\right)>0$ for any two zeros $x_{0}$ and $x_{1}$ of $P_{n+1}(x), n \geqslant 1$ (respectively, $W_{n}\left(y_{0}\right) W_{n}\left(y_{1}\right)>0$ for any two zeros $y_{0}$ and $y_{1}$ of $\left.P_{n+1}^{\prime}(x), n \geqslant 1\right)$, then $\left\{P_{n}(x)\right\}_{n=0}^{\infty}$ (respectively, $\left.\left\{P_{n}^{\prime}(x)\right\}_{n=1}^{\infty}\right)$ is a Sturm sequence in $I$.

Proof. Assume first that $W_{n}\left(x_{0}\right) W_{n}\left(x_{1}\right)>0$ for any two zeros $x_{0}$ and $x_{1}$ of $P_{n+1}(x), n \geqslant 1$. Since $P_{n+1}\left(x_{0}\right)=0, W_{n}\left(x_{0}\right)=P_{n}\left(x_{0}\right) P_{n+1}^{\prime}\left(x_{0}\right) \neq 0$ and so $P_{n+1}^{\prime}\left(x_{0}\right) \neq 0, n \geqslant 1$. Hence for all $n \geqslant 1$, zeros of $P_{n}(x)$ are simple. Let $a<x_{n 1}<x_{n 2}<\cdots<x_{n n}<b$ be the zeros of $P_{n}(x)$. We may and shall assume that all $P_{n}(x)$ are monic polynomials. Then

$$
\operatorname{sgn} P_{n}^{\prime}\left(x_{n k}\right)=(-1)^{n-k}, \quad 1 \leqslant k \leqslant n .
$$

On the other hand, we have by the assumption that

$$
\begin{aligned}
& W_{n}\left(x_{n+1, k}\right) W_{n}\left(x_{n+1, k+1}\right) \\
& =P_{n}\left(x_{n+1, k}\right) P_{n+1}^{\prime}\left(x_{n+1, k}\right) P_{n}\left(x_{n+1, k+1}\right) P_{n+1}^{\prime}\left(x_{n+1, k+1}\right)>0, \quad 1 \leqslant k \leqslant n .
\end{aligned}
$$


Hence by $(2.4)$

$$
P_{n}\left(x_{n+1, k}\right) P_{n}\left(x_{n+1, k+1}\right)<0, \quad 1 \leqslant k \leqslant n,
$$

so that $P_{n}(x)$ has one and only one zero in each interval $\left(x_{n+1, k}, x_{n+1, k+1}\right), 1 \leqslant$ $k \leqslant n$. Hence, $\left\{P_{n}(x)\right\}_{n=0}^{\infty}$ is a Sturm sequence in $I$.

We now assume that $W_{n}\left(y_{0}\right) W_{n}\left(y_{1}\right)>0$ for any two zeros $y_{0}$ and $y_{1}$ of $P_{n+1}^{\prime}(x)$, $n \geqslant 1$. Since $P_{n+1}^{\prime}\left(y_{0}\right)=0, W_{n}\left(y_{0}\right)=-P_{n}^{\prime}\left(y_{0}\right) P_{n+1}\left(y_{0}\right) \neq 0$ and so $P_{n+1}\left(y_{0}\right) \neq$ $0, n \geqslant 1$. Hence for all $\mathrm{n} \geqslant 1$, zeros of $P_{n}(x)$ are simple.

Let $a<x_{n 1}<x_{n 2}<\cdots<x_{n n}<b$ be the zeros of $P_{n}(x)$. Then by Rolle's theorem, $P_{n}^{\prime}(x)$ has one and only one zero $y_{n k}$ in each interval $\left(x_{n k}, x_{n, k+1}\right), 1 \leqslant$ $k \leqslant n-1$.

Assuming all $P_{n}(x)$ are monic, we have

$$
\operatorname{sgn} P_{n}\left(y_{n k}\right)=(-1)^{n-k}, \quad 1 \leqslant k \leqslant n-1 .
$$

On the other hand, we have by the assumption that

$$
\begin{aligned}
& W_{n}\left(y_{n+1, k}\right) W_{n}\left(y_{n+1, k+1}\right) \\
& =P_{n}^{\prime}\left(y_{n+1, k}\right) P_{n+1}\left(y_{n+1, k}\right) P_{n}^{\prime}\left(y_{n+1, k+1}\right) P_{n+1}\left(y_{n+1, k+1}\right)>0, \quad 1 \leqslant k \leqslant n-1 .
\end{aligned}
$$

Hence by $(2.5)$

$$
P_{n}^{\prime}\left(y_{n+1, k}\right) P_{n}^{\prime}\left(y_{n+1, k+1}\right)<0, \quad 1 \leqslant k \leqslant n-1,
$$

so that $P_{n}^{\prime}(x)$ has one and only one zero in each interval $\left(y_{n+1, k}, y_{n+1, k+1}\right), 1 \leqslant k \leqslant$ $n-1$. Hence, $\left\{P_{n}^{\prime}(x)\right\}_{n=1}^{\infty}$ is also a Sturm sequence in $I$.

Corollary 2.2. Let $\left\{P_{n}(x)\right\}_{n=0}^{\infty}$ be a PS such that all zeros of $P_{n}(x), n \geqslant 1$, are real and lie in $I=(a, b)$. If $W_{n}(x)>0, n \geqslant 1$, for all real $x$, then both $\left\{P_{n}(x)\right\}_{n=0}^{\infty}$ and $\left\{P_{n}^{\prime}(x)\right\}_{n=1}^{\infty}$ are Sturm sequences in $I$.

Remark. Since $W_{n}(x)$ is a monic polynomial of degree $2 n, W_{n}(x)>0$ for all real $x$ if and only if $W_{n}(x) \neq 0$ for all real $x$.

It is well known that if $\left\{P_{n}(x)\right\}_{n=0}^{\infty}$ is an OPS, then $W_{n}(x)>0, n \geqslant 1$, for all real $x$, which follows immediately from the Christoffel-Darboux identity satisfied by any TPS (see Chihara [2], Chapter 1.4). Therefore we have the following as a special case of Corollary 2.2 .

Theorem 2.3. If $\left\{P_{n}(x)\right\}_{n=0}^{\infty}$ is an OPS, then both $\left\{P_{n}(x)\right\}_{n=0}^{\infty}$ and $\left\{P_{n}^{\prime}(x)\right\}_{n=1}^{\infty}$ are Sturm sequences. Moreover, if $[a, b]$ is the true interval of orthogonality of $\left\{P_{n}(x)\right\}_{n=0}^{\infty}$, then $\left\{P_{n}(x)\right\}_{n=0}^{\infty}$ and $\left\{P_{n}^{\prime}(x)\right\}_{n=1}^{\infty}$ are Sturm sequences in $(a, b)$.

Proof. Since any OPS is a Sturm sequence and the true interval of orthogonality of $\left\{P_{n}(x)\right\}_{n=0}^{\infty}$ is the smallest closed interval containing all zeros of $P_{n}(x), n \geqslant 1$, the conclusion follows immediately from Corollary 2.2.

It is well known that for a PS $\left\{P_{n}(x)\right\}_{n=0}^{\infty}$, both $\left\{P_{n}(x)\right\}_{n=0}^{\infty}$ and $\left\{P_{n}^{\prime}(x)\right\}_{n=1}^{\infty}$ are TPS's (respectively, OPS's) if and only if $\left\{P_{n}(x)\right\}_{n=0}^{\infty}$ is a classical TPS (respectively, a classical OPS). This fact was first proved for OPS's by Sonine [9] and Hahn [3] and was later extended to TPS's (see [6]). For a simple unified proof of 
this fact and some other characterizations of classical orthogonal polynomials, we refer to $[8]$.

Hence, Theorem 2.3 answers negatively the question by Karlin and Szegö [5] : if $\left\{P_{n}(x)\right\}_{n=0}^{\infty}$ is an OPS and $\left\{P_{n}^{\prime}(x)\right\}_{n=1}^{\infty}$ is a Sturm sequence, is $\left\{P_{n}(x)\right\}_{n=0}^{\infty}$ a classical OPS?

We finally ask the following question: if $\left\{P_{n}(x)\right\}_{n=0}^{\infty}$ is a TPS but not an OPS, can $\left\{P_{n}(x)\right\}_{n=0}^{\infty}$ be a Sturm sequence?

Theorem 2.4. Let $\left\{P_{n}(x)\right\}_{n=0}^{\infty}$ be a TPS. Then $\left\{P_{n}(x)\right\}_{n=0}^{\infty}$ is a Sturm sequence if and only if $\left\{P_{n}(x)\right\}_{n=0}^{\infty}$ is an OPS.

Proof. The sufficiency follows from Theorem 2.3. We now assume that $\left\{P_{n}(x)\right\}_{n=0}^{\infty}$ is a Sturm sequence. By Farvard's theorem, $\left\{P_{n}(x)\right\}_{n=0}^{\infty}$ satisfies a three-term recurrence relation:

$$
P_{n+1}(x)=\left(x-b_{n}\right) P_{n}(x)-c_{n} P_{n-1}(x), \quad n \geqslant 0 \quad\left(P_{-1}(x) \equiv 0\right)
$$

(assuming all $P_{n}(x)$ are monic) where $c_{n} \neq 0, n \geqslant 1$. If we let $x_{n 1}<x_{n 2}<\cdots<$ $x_{n n}$ be the zeros of $P_{n}(x)$, then

$$
b_{n}=\sum_{k=1}^{n+1} x_{n+1, k}-\sum_{k=1}^{n} x_{n k}=x_{n+1,1}+\sum_{k=1}^{n}\left(x_{n+1, k+1}-x_{n k}\right) .
$$

Hence

$$
x_{n+1,1}-b_{n}=\sum_{k=1}^{n}\left(x_{n k}-x_{n+1, k+1}\right)<0
$$

since $x_{n+1, k}<x_{n k}<x_{n+1, k+1}, 1 \leqslant k \leqslant n$. From (2.6), we also have

$$
c_{n}=\frac{\left(x_{n+1,1}-b_{n}\right) P_{n}\left(x_{n+1,1}\right)}{P_{n-1}\left(x_{n+1,1}\right)}, \quad n \geqslant 1 .
$$

Since $P_{n}\left(x_{n+1,1}\right) P_{n-1}\left(x_{n+1,1}\right)<0, c_{n}>0, n \geqslant 1$, so that $\left\{P_{n}(x)\right\}_{n=0}^{\infty}$ is an OPS (see Chihara [2], Chapter 1.4).

In particular, Theorem 2.4 implies that if $\left\{P_{n}(x)\right\}_{n=0}^{\infty}$ is a TPS but not an OPS, then $\left\{P_{n}(x)\right\}_{n=0}^{\infty}$ cannot be a Sturm sequence. However, $\left\{P_{n}^{\prime}(x)\right\}_{n=1}^{\infty}$ may be a Sturm sequence as the next example shows.

Example. Consider Laguerre polynomials $\left\{L_{n}^{(\alpha)}(x)\right\}_{n=0}^{\infty}$ satisfying the secondorder differential equation

$$
x y^{\prime \prime}(x)+(\alpha+1-x) y^{\prime}(x)=-n y(x) .
$$

It is well known that $\left\{L_{n}^{(\alpha)}(x)\right\}_{n=0}^{\infty}$ is a TPS if and only if $\alpha \neq-1,-2, \ldots$ and is an OPS if and only if $\alpha>-1$. We also have (see Chihara [2], p.149)

$$
\frac{d}{d x} L_{n}^{(\alpha)}(x)=-L_{n-1}^{(\alpha+1)}(x), \quad n \geqslant 1 .
$$

Hence if $-2<\alpha<-1$, then $\left\{L_{n}^{(\alpha)}(x)\right\}_{n=0}^{\infty}$ is a TPS (but not an OPS) but $\left\{\frac{d}{d x} L_{n}^{(\alpha)}(x)\right\}_{n=1}^{\infty}$ is an OPS and so is a Sturm sequence. 


\section{ACKNOWLEDGMENTS}

This work is partially supported by Global Analysis Research Center at Seoul National University and Korea Ministry of Education (BSRI 1420).

\section{REFERENCES}

1. W. A. Al-Salam and T. S. Chihara, Another characterization of the classical orthogonal polynomials, SIAM J. Math. Anal. 3 (1972), 65-70. MR 47:5320

2. T. S. Chihara, An introduction to orthogonal polynomials, Gordon Breach, New York, 1977. MR 58:1979

3. W. Hahn, Über die Jacobischen Polynome und zwei verwandte Polynomklassen, Math. Z. 39 (1935), 634-638.

4. — Über höhere Ableitungen von Orthogonalpolynomen, Math. Z. 43 (1937), 101.

5. S. Karlin and G. Szegö, On certain determinants whose elements are orthogonal polynomials, J. Analyse Math. 8 (1961), 1-157. MR 26:539

6. H. L. Krall, On derivatives of orthogonal polynomials, Bull. Amer. Math. Soc 42 (1936), 423-428.

7. $867-870$.

8. K. H. Kwon, J. K. Lee, and B. H. Yoo, Characterizations of classical orthogonal polynomials, Results in Math. 24 (1993), 119-128. MR 94i:33011

9. N. J. Sonine, Recherches sur les fonctions cylindrigues et le développment des fonctions continues en series, Math. Ann 16 (1880), 1-80.

Department of Mathematics, Korea Advanced Institute of Science and Technology, 373-1 Kusong-Dong, Yusong-Ku, TAejon 305-701, Korea

E-mail address: khkwon@jacobi.kaist.ac.kr 